\title{
On the smallest pseudopower
}

by

Jean Bourgain (Princeton, NJ), Sergei V. Konyagin (Moscow), Carl Pomerance (Hanover, NH) and Igor E. ShParlinski (Sydney)

1. Introduction. Let $g$ be a fixed integer with $|g| \geq 2$. Following E. Bach, R. Lukes, J. Shallit and H. C. Williams [1], we say that an integer $n>0$ is an $x$-pseudopower to base $g$ if $n$ is not a power of $g$ over the integers but is a power of $g$ modulo all primes $p \leq x$, that is, if for all primes $p \leq x$ there exists an integer $e_{p} \geq 0$ such that $n \equiv g^{e_{p}}(\bmod p)$.

Denote by $q_{g}(x)$ the least $x$-pseudopower to base $g$.

A well-known result of A. Schinzel [8] asserts that if $f$ and $g>0$ are integers such that $f \neq g^{k}$ for all integers $k \geq 0$, then for infinitely many primes $p$ the congruence $g^{k} \equiv f(\bmod p)$ does not have solutions in nonnegative integers $k$. Therefore,

$$
q_{g}(x) \rightarrow \infty \quad \text { as } x \rightarrow \infty .
$$

E. Bach, R. Lukes, J. Shallit and H. C. Williams [1] have shown that if the Riemann Hypothesis holds for Dedekind zeta functions, then there is a constant $A_{g}>0$ such that

$$
q_{g}(x) \geq \exp \left(A_{g} \sqrt{x} /(\log x)^{2}\right) .
$$

On the other hand, if

$$
M_{x}=\prod_{p \leq x} p
$$

is the product of all primes $p \leq x$, then $q_{g}(x) \leq 2 M_{x}+1$ when $x \geq 2$. Since, by the prime number theorem, $M_{x}=\exp (x+o(x))$, we have

$$
q_{g}(x) \leq \exp ((1+o(1)) x) \quad \text { as } x \rightarrow \infty .
$$

Supported by numerical data, a heuristic argument is given in [1] suggesting that $q_{g}(x)$ for fixed $g$ is about $\exp \left(c_{g} x / \log x\right)$, where $c_{g}>0$. In [7], towards this conjecture, the upper bound

2010 Mathematics Subject Classification: 11A07, 11L07.

Key words and phrases: pseudopowers, exponential sums, multiplicative subgroups of residue rings. 


$$
q_{g}(x) \leq \exp \left(c_{g} \frac{x \log \log x}{\log x}\right)
$$

is proved conditionally under the Extended Riemann Hypothesis.

In [5], combining some bounds of exponential sums with new results about the average behaviour of the multiplicative order of $g$ modulo prime numbers, the bound (2) has been improved as

$$
q_{g}(x) \leq \exp (0.88715 x)
$$

for $x$ sufficiently large and $|g| \leq x$. Here we obtain a further improvement.

THEOREM 1. For all sufficiently large numbers $x$ and all integers $g$ with $1<|g| \leq x$, we have

$$
q_{g}(x) \leq \exp (0.86092 x) .
$$

The result is based on a combination of the approach of [5] with some new estimates on the distribution of multiplicative subgroups in residue rings, which in turn are based on the results and ideas from [2].

We remark that [5] and [7] give some results showing some level of uniform distribution for $x$-pseudopowers to base $g$, unconditionally and under the Extended Riemann Hypothesis, respectively. Unfortunately, it seems that our approach here does not imply results on uniform distribution; it remains an open problem to improve the estimates of [5] and [7].

2. Preliminaries. For an integer $m$ we use $\mathbb{Z}_{m}$ to denote the residue ring modulo $m$ and we also use $\mathbb{Z}_{m}^{*}$ to denote the group of units of $\mathbb{Z}_{m}$.

Let $\mathcal{G}$ be a multiplicative subgroup of $\mathbb{Z}_{m}^{*}$ of order $t$. We denote by $H_{m}(\mathcal{G})$ the largest gap between the elements of $\mathcal{G}$, that is,

$$
H_{m}(\mathcal{G})=\max \left\{H: \exists u \in \mathbb{Z}_{m} \text { such that } u+j \notin \mathcal{G}, j=1, \ldots, H\right\} .
$$

For a prime $p$ with $\operatorname{gcd}(g, p)=1$, we denote by $\mathcal{G}_{g, p}$ the subgroup of $\mathbb{Z}_{p}^{*}$ generated by powers of $g$ modulo $p$, that is,

$$
\mathcal{G}_{g, p}=\left\{n \in \mathbb{Z}_{p}: n \equiv g^{k}(\bmod p) \text { for some nonnegative } k \in \mathbb{Z}\right\} .
$$

Clearly, if $\operatorname{gcd}(g, p)=1$ then $\mathcal{G}_{g, p}$ is a subgroup of $\mathbb{Z}_{p}^{*}$. Finally, if $p \mid g$, then we define $\mathcal{G}_{g, p}=\{1\}$.

We consider the subgroup of $\mathbb{Z}_{M_{x}}^{*}$ defined by

$$
\mathcal{G}_{g}(x)=\left\{n \in\left[0, M_{x}\right): n \in \mathcal{G}_{g, p} \text { for all primes } p \leq x\right\} .
$$

Since we are assuming that $|g| \leq x$, we note that $\mathcal{G}_{g}(x)$ consists of both the $x$-pseudopowers to base $g$ in $\left[0, M_{x}\right)$ that are coprime to $M_{x}$ and the number 1 . Thus, the interval $\left[2, H_{M_{x}}\left(\mathcal{G}_{g}(x)\right)+2\right]$ contains at least one $x$ pseudopower to the base $g$ and we deduce that

$$
q_{g}(x) \leq H_{M_{x}}\left(\mathcal{G}_{g}(x)\right)+2 .
$$

Therefore we concentrate on getting an upper bound on $H_{M_{x}}\left(\mathcal{G}_{g}(x)\right)$. 
3. Gaps between elements of multiplicative subgroups of residue rings and exponential sums. We need an analogue of [6, Lemma 7.1] which relates $H_{m}(\mathcal{G})$ with certain exponential sums.

Given a subgroup $\mathcal{G}$ of $\mathbb{Z}_{m}^{*}$, we denote by $M_{\lambda}(m, \mathcal{G} ; h)$ the number of solutions to the congruence

$$
\lambda \equiv a w(\bmod m), \quad 1 \leq|a| \leq h, w \in \mathcal{G} .
$$

Essentially, $M_{\lambda}(m, \mathcal{G} ; h)$ is the number of nonzero elements of the set $\lambda \mathcal{G}$ that lie in the interval $[-h, h]$. (Note that $\lambda$ need not be coprime to $m$, so that the translated subgroup $\lambda \mathcal{G}$ need not be a coset in $\mathbb{Z}_{m}^{*}$.)

Also, we put

$$
\mathbf{e}_{m}(z)=\exp (2 \pi i z / m)
$$

and define exponential sums

$$
S_{\lambda}(m, \mathcal{G})=\sum_{v \in \mathcal{G}} \mathbf{e}_{m}(\lambda v)
$$

Lemma 2. Assume that $\mathcal{G}$ is of order $t$ and that for some positive integer $h \leq m / 2$ we have

$$
\sum_{\lambda \in \mathbb{Z}_{m}} M_{\lambda}(m, \mathcal{G} ; h)\left|S_{\lambda}(m, \mathcal{G})\right| \leq 0.5 t^{2}
$$

Then, as $m \rightarrow \infty$,

$$
H_{m}(\mathcal{G}) \leq m^{1+o(1)} h^{-1}
$$

Proof. Let us fix some $\varepsilon>0$. We put

$$
s=\left\lceil 0.5\left(1+\varepsilon^{-1}\right)\right\rceil, \quad Z=\left\lceil m^{1+\varepsilon} h^{-1}\right\rceil .
$$

Obviously, it is enough to show that for a sufficiently large $m$ and any integer $U$ the congruence

$$
\begin{aligned}
v \equiv U+x_{1}+\cdots+x_{s}-y_{1}-\cdots-y_{s}(\bmod m), & \\
& v \in \mathcal{G}, 0 \leq x_{1}, y_{1}, \ldots, x_{s}, y_{s}<Z,
\end{aligned}
$$

is solvable. Indeed, in this case we have $H_{m}(\mathcal{G}) \leq 2 s(Z-1)$ and since $\varepsilon>0$ is arbitrary the result follows.

For the number $Q$ of solutions to the congruence (5) one easily sees from the identity

$$
\frac{1}{m} \sum_{-(m-1) / 2 \leq a \leq m / 2} \mathbf{e}_{m}(a z)= \begin{cases}1 & \text { if } z \equiv 0(\bmod m) \\ 0 & \text { otherwise }\end{cases}
$$


which holds for any $z \in \mathbb{Z}$, that

$$
\begin{aligned}
Q= & \sum_{v \in \mathcal{G}} \sum_{0 \leq x_{1}, y_{1}, \ldots, x_{s}, y_{s}<Z} \frac{1}{m} \mathbf{e}_{m}\left(a\left(v-U-x_{1}-\cdots-x_{s}+y_{1}+\cdots+y_{s}\right)\right) \\
& \times \sum_{-(m-1) / 2 \leq a \leq m / 2} \mathbf{e}_{m}(-a U) \sum_{v \in \mathcal{G}} \mathbf{e}_{m}(a v) \\
= & \sum_{-(m-1) / 2 \leq a \leq m / 2} \sum_{0 \leq x_{1}, y_{1}, \ldots, x_{s}, y_{s}<Z} \mathbf{e}_{m}\left(-a\left(x_{1}+\cdots+x_{s}-y_{1}-\cdots-y_{s}\right)\right) \\
= & \frac{1}{m} \sum_{-(m-1) / 2 \leq a \leq m / 2} \mathbf{e}_{m}(-a U)\left|\sum_{0 \leq x<Z} \mathbf{e}_{m}(a x)\right|^{2 s} \sum_{v \in \mathcal{G}} \mathbf{e}_{m}(a v) .
\end{aligned}
$$

Therefore

$$
Q \geq t Z^{2 s} m^{-1}-\sigma_{1} m^{-1}-\sigma_{2} m^{-1},
$$

where

$$
\begin{aligned}
& \sigma_{1}=\sum_{1 \leq|a| \leq h}\left|\sum_{0 \leq x<Z} \mathbf{e}_{m}(a x)\right|^{2 s}\left|\sum_{v \in \mathcal{G}} \mathbf{e}_{m}(a v)\right|, \\
& \sigma_{2}=\sum_{h<|a| \leq m / 2}\left|\sum_{0 \leq x<Z} \mathbf{e}_{m}(a x)\right|^{2 s}\left|\sum_{v \in \mathcal{G}} \mathbf{e}_{m}(a v)\right| .
\end{aligned}
$$

For $1 \leq|a| \leq h$ we use the trivial estimate

$$
\left|\sum_{0 \leq x<Z} \mathbf{e}_{m}(a x)\right| \leq Z
$$

and derive

$$
\begin{aligned}
\sigma_{1} & \leq Z^{2 s} \sum_{1 \leq|a| \leq h}\left|\sum_{v \in \mathcal{G}} \mathbf{e}_{m}(a v)\right|=\frac{Z^{2 s}}{\# \mathcal{G}} \sum_{1 \leq|a| \leq h} \sum_{w \in \mathcal{G}}\left|\sum_{v \in \mathcal{G}} \mathbf{e}_{m}(a w v)\right| \\
& =\frac{Z^{2 s}}{\# \mathcal{G}} \sum_{\lambda \in \mathbb{Z}_{m}} M_{\lambda}(m, \mathcal{G} ; h)\left|S_{\lambda}(m, \mathcal{G})\right| .
\end{aligned}
$$

Therefore, by the conditions of the lemma, we have

$$
\sigma_{1} \leq 0.5 t Z^{2 s} .
$$

If $h<|a| \leq m / 2$ then we use the bound

$$
\left|\sum_{0 \leq x<Z} \mathbf{e}_{m}(a x)\right| \ll \frac{m}{|a|}
$$


(see [4, bound (8.6)]). From the trivial bound

$$
\left|\sum_{v \in \mathcal{G}} \mathbf{e}_{m}(a v)\right| \leq t
$$

recalling the choice of $Z$, we obtain

$$
\sigma_{2} \ll \sum_{h<|a| \leq m / 2}\left(\frac{m}{|a|}\right)^{2 s} t \ll t \frac{m^{2 s}}{h^{2 s-1}} \leq t \frac{Z^{2 s} h}{m^{2 s \varepsilon}} \ll t \frac{Z^{2 s} h}{m^{1+\varepsilon}}
$$

as $2 s \varepsilon>1+\varepsilon$ for the above choice of $s$. In particular,

$$
\sigma_{2} \ll t Z^{2 s} m^{-\varepsilon}
$$

Substituting (7) and (8) in (6), we obtain

$$
Q \geq 0.5 t Z^{2 s} m^{-1}+O\left(t Z^{2 s} m^{-1-\varepsilon}\right) .
$$

Thus $Q>0$ provided that $m$ is large enough, and the result follows.

4. Further preparations. Now, for each $d \mid m$, we collect together the terms in the sum in Lemma 2 with $\operatorname{gcd}(\lambda, m)=d$.

In particular, let $\mathcal{G}_{d}$ be the homomorphic image of $\mathcal{G}$ in $\mathbb{Z}_{m / d}^{*}$. It is easy to verify that every element of $\mathcal{G}$ is mapped to

$$
\#\{w \in \mathcal{G}: w \equiv 1(\bmod m / d)\}=\frac{\# \mathcal{G}}{\# \mathcal{G}_{d}}
$$

elements of $\mathcal{G}_{d}$. Thus,

$$
\begin{aligned}
\sum_{\lambda \in \mathbb{Z}_{m}} M_{\lambda}(m, \mathcal{G} ; h)\left|S_{\lambda}(m, \mathcal{G})\right|=\sum_{d \mid m} \sum_{\substack{\lambda \in \mathbb{Z}_{m} \\
\operatorname{gcd}(\lambda, m)=d}} M_{\lambda}(m, \mathcal{G} ; h)\left|S_{\lambda}(m, \mathcal{G})\right| \\
=\sum_{d \mid m}\left(\frac{\# \mathcal{G}}{\# \mathcal{G}_{d}}\right)^{2} \sum_{\lambda \in \mathbb{Z}_{m / d}^{*}} M_{\lambda}\left(m / d, \mathcal{G}_{d} ; h / d\right)\left|S_{\lambda}\left(m / d, \mathcal{G}_{d}\right)\right|
\end{aligned}
$$

We remark that by the Hölder inequality

$$
\begin{aligned}
& \sum_{\lambda \in \mathbb{Z}_{m / d}^{*}} M_{\lambda}\left(m / d, \mathcal{G}_{d} ; h / d\right)\left|S_{\lambda}\left(m / d, \mathcal{G}_{d}\right)\right| \\
& =\sum_{\lambda \in \mathbb{Z}_{m / d}^{*}} M_{\lambda}\left(m / d, \mathcal{G}_{d} ; h / d\right)^{1 / 2}\left(M_{\lambda}\left(m / d, \mathcal{G}_{d} ; h / d\right)^{2}\right)^{1 / 4}\left(\left|S_{\lambda}\left(m / d, \mathcal{G}_{d}\right)\right|^{4}\right)^{1 / 4} \\
& \leq\left(\sum_{\lambda \in \mathbb{Z}_{m / d}^{*}} M_{\lambda}\left(m / d, \mathcal{G}_{d} ; h / d\right)\right)^{1 / 2}\left(\sum_{\lambda \in \mathbb{Z}_{m / d}^{*}} M_{\lambda}\left(m / d, \mathcal{G}_{d} ; h / d\right)^{2}\right)^{1 / 4} \\
& \quad \times\left(\sum_{\lambda \in \mathbb{Z}_{m / d}^{*}}\left|S_{\lambda}\left(m / d, \mathcal{G}_{d}\right)\right|^{4}\right)^{1 / 4} .
\end{aligned}
$$


Clearly,

$$
\sum_{\lambda \in \mathbb{Z}_{m / d}^{*}} M_{\lambda}\left(m / d, \mathcal{G}_{d} ; h / d\right) \leq \sum_{\lambda \in \mathbb{Z}_{m / d}} M_{\lambda}\left(m / d, \mathcal{G}_{d} ; h / d\right) \leq 2 h \# \mathcal{G}_{d} / d .
$$

Given a multiplicative subgroup $\mathcal{H} \subseteq \mathbb{Z}_{n}^{*}$ in the residue ring modulo a positive integer $n$, and a positive integer $h$, we define

$$
\begin{array}{r}
V(n, \mathcal{H} ; h)=\#\left\{\left(u_{1}, u_{2}, v\right): u_{1}, u_{2} \in[-h, h], \operatorname{gcd}\left(u_{1} u_{2}, n\right)=1,\right. \\
\left.v \in \mathcal{H}, u_{1} v \equiv u_{2}(\bmod n)\right\} .
\end{array}
$$

We have

$$
\begin{aligned}
\sum_{\lambda \in \mathbb{Z}_{m / d}^{*}} M_{\lambda}\left(m / d, \mathcal{G}_{d} ; h / d\right)^{2} & \\
\leq & \sum_{\lambda \in \mathbb{Z}_{m / d}^{*}} \#\left\{u_{1}, u_{2} \in[-h / d, h / d]: u_{1}, u_{2} \in \lambda \mathcal{G}_{d}\right\} \\
& =\#\left\{\left(u_{1}, u_{2}, v_{1}, v_{2}\right): u_{1}, u_{2} \in[-h / d, h / d], \operatorname{gcd}\left(u_{1} u_{2}, m / d\right)=1,\right. \\
& \left.v_{1}, v_{2} \in \mathcal{G}_{d}, u_{1} v_{1} \equiv u_{2} v_{2}(\bmod m / d)\right\} \\
& =\# \mathcal{G}_{d} V\left(m / d, \mathcal{G}_{d} ; h / d\right) .
\end{aligned}
$$

Therefore,

$$
\begin{aligned}
& \sum_{\lambda \in \mathbb{Z}_{m / d}^{*}} M_{\lambda}\left(m / d, \mathcal{G}_{d} ; h / d\right)\left|S_{\lambda}\left(m / d, \mathcal{G}_{d}\right)\right| \\
& \quad \leq 2^{1 / 2} h^{1 / 2} d^{-1 / 2}\left(\# \mathcal{G}_{d}\right)^{3 / 4} V\left(m / d, \mathcal{G}_{d} ; h / d\right)^{1 / 4} W_{4}\left(m / d, \mathcal{G}_{d}\right)^{1 / 4}
\end{aligned}
$$

where

$$
W_{4}\left(m / d, \mathcal{G}_{d}\right)=\sum_{\lambda \in \mathbb{Z}_{m / d}^{*}}\left|S_{\lambda}\left(m / d, \mathcal{G}_{d}\right)\right|^{4} .
$$

For $V\left(m / d, \mathcal{G}_{d} ; h / d\right)$ we use the bound which is readily available from [2].

For the fourth moment $W_{4}\left(m / d, \mathcal{G}_{d}\right)$ such general purpose bounds are not available. However, in the case of our interest, that is, for the modulus $m=$ $M_{x}$ (given by (1)) and the subgroup $\mathcal{G}_{g}(x)$ (given by $(3)$ ), we obtain such a bound using some results from [3] and [5]. Substituting these estimates in (11) enables us to show that the condition of Lemma 2 is satisfied for a sufficiently large $h$, which in turn leads to the desired estimate on $H_{m}(\mathcal{G})$.

5. Bound on $V(n, \mathcal{G} ; h)$. We recall the following result of [2, Lemma 4], which gives the desired estimate on $V(n, \mathcal{H} ; h)$, defined by (10) for an arbitrary modulus $n \geq 1$ and a subgroup $\mathcal{H} \subseteq \mathbb{Z}_{n}^{*}$.

LEMMA 3. Let $\nu \geq 1$ be a fixed integer and let $n \rightarrow \infty$. Assume that $\mathcal{H}$ is a multiplicative subgroup of $\mathbb{Z}_{n}^{*}$. Then for any positive number $h \leq n$, we 
have

$$
V(n, \mathcal{H}, h) \leq h T^{\frac{2 \nu+1}{2 \nu(\nu+1)}} n^{-\frac{1}{2(\nu+1)}+o(1)}+h^{2} T^{1 / \nu} n^{-1 / \nu+o(1)}
$$

as $n \rightarrow \infty$, where

$$
T=\max \left\{\# \mathcal{H}, n^{1 / 2}\right\} .
$$

6. Bounds on the fourth moment of exponential sums. For $d \mid M_{x}$, we consider the homomorphic image of $\mathcal{G}_{g}(x)$ in $\mathbb{Z}_{M_{x} / d}^{*}$, which we denote by $\mathcal{G}_{g}(d ; x)$ (this slightly deviates from our previous notation $\mathcal{G}_{g}(x)_{d}$, which for typographical reasons, we prefer to avoid).

As in [5] we remark that by the Chinese remainder theorem we have

$$
S_{\lambda}\left(M_{x} / d, \mathcal{G}_{g}(d ; x)\right)=\sum_{v \in \mathcal{G}_{g}(d ; x)} \mathbf{e}_{m}(\lambda v)=\prod_{\substack{p \leq x \\ \operatorname{gcd}(p, d)=1}} \sum_{v \in \mathcal{G}_{g, p}} \mathbf{e}_{p}\left(\lambda_{p} v\right),
$$

where $\lambda_{p} \in \mathbb{Z}_{p}$ is determined by the condition

$$
\lambda_{p}\left(M_{x} / p\right) \equiv \lambda\left(\bmod M_{x}\right) .
$$

We also remark that when $\lambda$ runs through $\mathbb{Z}_{M_{x} / d}$, the corresponding vector $\left(\lambda_{p}\right)_{p \leq x, p \nmid d}$ runs through the Cartesian product

$$
\mathcal{U}_{x}(d)=\prod_{\substack{p \leq x \\ \operatorname{gcd}(p, d)=1}} \mathbb{Z}_{p}^{*}
$$

Thus, using (12), we obtain

$$
\begin{aligned}
W_{4}\left(M_{x} / d, \mathcal{G}_{g}(d ; x)\right) & =\sum_{\lambda \in \mathbb{Z}_{M_{x} / d}^{*}}\left|S_{\lambda}\left(M_{x} / d, \mathcal{G}_{g}(d ; x)\right)\right|^{4} \\
& =\sum_{\left(\lambda_{p}\right)_{p \leq x, \operatorname{gcd}(p, d)=1} \in \mathcal{U}_{x}(d)} \prod_{\substack{p \leq x \\
\operatorname{gcd}(p, d)=1}}\left|\sum_{v \in \mathcal{G}_{g, p}} \mathbf{e}_{p}\left(\lambda_{p} v\right)\right|^{4} .
\end{aligned}
$$

Therefore

$$
W_{4}\left(M_{x} / d, \mathcal{G}_{g}(d ; x)\right)=\prod_{\substack{p \leq x \\ \operatorname{gcd}(p, d)=1}} \sum_{\lambda_{p} \in \mathbb{Z}_{p}^{*}}\left|\sum_{v \in \mathcal{G}_{g, p}} \mathbf{e}_{p}\left(\lambda_{p} v\right)\right|^{4} .
$$

We now recall the bound of [3, Lemma 3] on the fourth moment of exponential sums over multiplicative subgroups in a residue ring modulo a prime (see also [6, Lemma 3.3]).

Lemma 4. For any prime $p$ and subgroup $\mathcal{G}$ of $\mathbb{Z}_{p}^{*}$ of order $\# \mathcal{G}=t<$ $p^{2 / 3}$, the following bound holds:

$$
\sum_{\lambda \in \mathbb{Z}_{p}^{*}}\left|\sum_{v \in \mathcal{G}} \mathbf{e}_{p}(\lambda v)\right|^{4} \ll p t^{5 / 2}
$$


Proof. It is enough to note that by the orthogonality of exponential functions

$$
\begin{aligned}
\sum_{\lambda \in \mathbb{Z}_{p}^{*}}\left|\sum_{v \in \mathcal{G}} \mathbf{e}_{p}(\lambda v)\right|^{4} & \leq \sum_{\lambda \in \mathbb{Z}_{p}}\left|\sum_{v \in \mathcal{G}} \mathbf{e}_{p}(\lambda v)\right|^{4} \\
& =p \#\left\{v_{1}+v_{2}=v_{3}+v_{4}: v_{1}, v_{2}, v_{3}, v_{4} \in \mathcal{G}\right\}
\end{aligned}
$$

and then apply the bound of [3, Lemma 3].

For groups of order $\# \mathcal{G}=t>p^{2 / 3}$ we use a different bound which relies on some classical estimates.

Lemma 5. For any prime $p$ and subgroup $\mathcal{G}$ of $\mathbb{Z}_{p}^{*}$ of order $\# \mathcal{G}=t \geq$ $p^{2 / 3}$, the following bound holds:

$$
\sum_{\lambda \in \mathbb{Z}_{p}^{*}}\left|\sum_{v \in \mathcal{G}} \mathbf{e}_{p}(\lambda v)\right|^{4} \leq p^{2} t .
$$

Proof. We recall the well-known estimate

$$
\left|\sum_{v \in \mathcal{G}} \mathbf{e}_{p}(\lambda v)\right| \leq p^{1 / 2}
$$

for any $t$ and $\lambda \in \mathbb{Z}_{p}^{*}$ (see [6, Theorem 3.4]). Therefore

$$
\sum_{\lambda \in \mathbb{Z}_{p}^{*}}\left|\sum_{v \in \mathcal{G}} \mathbf{e}_{p}(\lambda v)\right|^{4} \leq p \sum_{\lambda \in \mathbb{Z}_{p}}\left|\sum_{v \in \mathcal{G}} \mathbf{e}_{p}(\lambda v)\right|^{2}=p \sum_{\lambda \in \mathbb{Z}_{p}} \sum_{v_{1}, v_{2} \in \mathcal{G}} \mathbf{e}_{p}\left(\lambda\left(v_{1}-v_{2}\right)\right)=p^{2} t,
$$

as after the change of the order of summation, the sum over $\lambda$ vanishes if $v_{1} \neq v_{2}$ and is equal to $p$ otherwise.

For a prime $p \nmid g$ we denote by $t_{g, p}=\# \mathcal{G}_{g, p}$ the multiplicative order of $g$ modulo $p$. We also put $t_{g, p}=1$ for $p \mid g$. In particular,

$$
\# \mathcal{G}_{g}(d ; x)=\prod_{\substack{p \leq x \\ \operatorname{gcd}(p, d)=1}} t_{g, p}
$$

We also put

$$
Q_{g}(d ; x)=\prod_{\substack{p \leq x \\ \operatorname{gcd}(p, d)=1 \\ t_{g, p} \geq p^{2 / 3}}}\left(t_{g, p} p^{-2 / 3}\right) .
$$

We are now ready to obtain the desired estimate of $W_{4}\left(M_{x}, \mathcal{G}_{g}(x)\right)$.

Lemma 6. We have

$$
W_{4}\left(M_{x} / d, \mathcal{G}_{g}(d ; x)\right) \ll \frac{M_{x}}{d}\left(\# \mathcal{G}_{g}(d ; x)\right)^{5 / 2} Q_{g}(d ; x)^{-3 / 2} .
$$


Proof. Substituting the bound of Lemmas 4 and 5 in (13), we see that

$$
\begin{aligned}
W_{4}\left(M_{x} / d, \mathcal{G}_{g}(d ; x)\right) \ll & \prod_{\substack{p \leq x \\
\operatorname{gcd}(p, d)=1 \\
t_{g, p}<p^{2 / 3}}}\left(p t_{g, p}^{5 / 2}\right) \prod_{\substack{p \leq x \\
\operatorname{gcd}(p, d)=1 \\
t_{g, p} \geq p^{2 / 3}}}\left(p^{2} t_{g, p}\right) \\
& =\prod_{\substack{p \leq x \\
\operatorname{gcd}(p, d)=1}}\left(p t_{g, p}^{5 / 2}\right) \prod_{\substack{p \leq x \\
\operatorname{gcd}(p, d)=1 \\
t_{g, p} \geq p^{2 / 3}}}\left(p t_{g, p}^{-3 / 2}\right),
\end{aligned}
$$

which implies the desired estimate.

7. Bounds on multiplicative orders. We recall the following two estimates, which are [5, Theorem 1] and [5, Lemma 9], respectively.

Lemma 7 . For $x$ sufficiently large, we have

$$
\# \mathcal{G}_{g}(x) \geq \exp (0.58045 x)
$$

uniformly for $1<|g| \leq x$.

Let

$$
Q_{g}(x)=\prod_{\substack{p \leq x \\ t_{g, p} \geq p^{2 / 3}}}\left(t_{g, p} p^{-2 / 3}\right) .
$$

Lemma 8. For $x$ sufficiently large, we have

$$
Q_{g}(x) \geq \exp (0.000217 x)
$$

uniformly for $1<|g| \leq x$.

8. Concluding the proof of Theorem 1. We now define

$$
T_{g}(d ; x)=\max \left\{\# \mathcal{G}_{g}(d ; x),\left(M_{x} / d\right)^{1 / 2}\right\} .
$$

Using Lemmas 3 and 6 together with (11), we obtain

$$
\begin{aligned}
& \sum_{\lambda \in \mathbb{Z}_{M_{x} / d}^{*}} M_{\lambda}\left(M_{x} / d, \mathcal{G}_{d} ; h / d\right)\left|S_{\lambda}\left(M_{x} / d, \mathcal{G}_{d}\right)\right| \\
\ll & h^{1 / 2} d^{-1 / 2}\left(\# \mathcal{G}_{g}(d ; x)\right)^{3 / 4} \\
& \times\left(h T_{g}(d ; x)^{\frac{2 \nu+1}{2 \nu(\nu+1)}}\left(\frac{M_{x}}{d}\right)^{-\frac{1}{2(\nu+1)}+o(1)}+h^{2} T_{g}(d ; x)^{1 / \nu}\left(\frac{M_{x}}{d}\right)^{-1 / \nu+o(1)}\right)^{1 / 4} \\
& \times\left(\frac{M_{x}}{d}\left(\# \mathcal{G}_{g}(d ; x)\right)^{5 / 2} Q_{g}(d ; x)^{-3 / 2}\right)^{1 / 4}
\end{aligned}
$$


$\ll h^{1 / 2}\left(\# \mathcal{G}_{g}(d ; x)\right)^{11 / 8} M_{x}^{1 / 4} d^{-3 / 4} Q_{g}(d ; x)^{-3 / 8}$

$\times\left(h T_{g}(d ; x)^{\frac{2 \nu+1}{2 \nu(\nu+1)}}\left(\frac{M_{x}}{d}\right)^{-\frac{1}{2(\nu+1)}+o(1)}+h^{2} T_{g}(d ; x)^{1 / \nu}\left(\frac{M_{x}}{d}\right)^{-1 / \nu+o(1)}\right)^{1 / 4}$.

Recalling (9), we now derive

$$
\sum_{\lambda \in \mathbb{Z}_{M_{x}}} M_{\lambda}\left(M_{x}, \mathcal{G} ; h\right)\left|S_{\lambda}\left(M_{x}, \mathcal{G}\right)\right| \leq\left(\# \mathcal{G}_{g}(x)\right)^{2} \sum_{d \mid M_{x}}\left(I_{g}(d ; x)+J_{g}(d ; x)\right),
$$

where

$$
\begin{aligned}
I_{g}(d ; x)= & h^{3 / 4}\left(\# \mathcal{G}_{g}(d ; x)\right)^{-5 / 8} Q_{g}(d ; x)^{-3 / 8} T_{g}(d ; x)^{\frac{2 \nu+1}{8 \nu(\nu+1)}} \\
& \times M_{x}^{\frac{2 \nu+1}{8(\nu+1)}+o(1)} d^{-\frac{6 \nu+5}{8(\nu+1)}}, \\
J_{g}(d ; x)= & h\left(\# \mathcal{G}_{g}(d ; x)\right)^{-5 / 8} Q_{g}(d ; x)^{-3 / 8} T_{g}(d ; x)^{\frac{1}{4 \nu}} M_{x}^{\frac{\nu-1}{4 \nu}+o(1)} d^{-\frac{3 \nu-1}{4 \nu}} .
\end{aligned}
$$

Therefore, using $T_{g}(d ; x) \leq \# \mathcal{G}_{g}(d ; x)+\left(M_{x} / d\right)^{1 / 2}$, we have

$$
I_{g}(d ; x) \leq A_{g}(d ; x)+B_{g}(d ; x), \quad J_{g}(d ; x) \leq C_{g}(d ; x)+D_{g}(d ; x),
$$

where

$$
\begin{aligned}
& A_{g}(d ; x)=h^{3 / 4}\left(\# \mathcal{G}_{g}(d ; x)\right)^{-\frac{5 \nu^{2}+3 \nu-1}{8 \nu(\nu+1)}} Q_{g}(d ; x)^{-3 / 8} M_{x}^{\frac{2 \nu+1}{8(\nu+1)}+o(1)} d^{-\frac{6 \nu+5}{8(\nu+1)}}, \\
& B_{g}(d ; x)=h^{3 / 4}\left(\# \mathcal{G}_{g}(d ; x)\right)^{-5 / 8} Q_{g}(d ; x)^{-3 / 8} M_{x}^{\frac{(2 \nu+1)^{2}}{16 \nu(\nu+1)}+o(1)} d^{-\frac{12 \nu^{2}+12 \nu+1}{16 \nu(\nu+1)}}, \\
& C_{g}(d ; x)=h\left(\# \mathcal{G}_{g}(d ; x)\right)^{-\frac{5 \nu-2}{8 \nu}} Q_{g}(d ; x)^{-3 / 8} M_{x}^{\frac{\nu-1}{4 \nu}+o(1)} d^{-\frac{3 \nu-1}{4 \nu}} \\
& D_{g}(d ; x)=h\left(\# \mathcal{G}_{g}(d ; x)\right)^{-5 / 8} Q_{g}(d ; x)^{-3 / 8} M_{x}^{\frac{2 \nu-1}{8 \nu}+o(1)} d^{-\frac{6 \nu-1}{8 \nu}} .
\end{aligned}
$$

We note that

$$
\# \mathcal{G}_{g}(d ; x) \geq \# \mathcal{G}_{g}(x) / d
$$

and also that

$$
Q_{g}(d ; x)=Q_{g}(x) \prod_{\substack{p \mid d \\ t_{g, p} \geq p^{2 / 3}}}\left(t_{g, p} p^{-2 / 3}\right)^{-1} \geq Q_{g}(x) / d^{1 / 3} .
$$

Therefore,

$$
\begin{aligned}
& A_{g}(d ; x) \leq h^{3 / 4}\left(\# \mathcal{G}_{g}(x)\right)^{-\frac{5 \nu^{2}+3 \nu-1}{8 \nu(\nu+1)}} Q_{g}(x)^{-3 / 8} M_{x}^{\frac{2 \nu+1}{8 \nu+1)}+o(1)} d^{-\frac{1}{8 \nu}} \\
& B_{g}(d ; x) \leq h^{3 / 4}\left(\# \mathcal{G}_{g}(x)\right)^{-5 / 8} Q_{g}(x)^{-3 / 8} M_{x}^{\frac{(2 \nu+1)^{2}}{16(\nu+1)}+o(1)} d^{-\frac{1}{16 \nu(\nu+1)}} \\
& C_{g}(d ; x) \leq h\left(\# \mathcal{G}_{g}(x)\right)^{-\frac{5 \nu-2}{8 \nu}} Q_{g}(x)^{-3 / 8} M_{x}^{\frac{\nu-1}{4 \nu}+o(1)} \\
& D_{g}(d ; x) \leq h\left(\# \mathcal{G}_{g}(x)\right)^{-5 / 8} Q_{g}(x)^{-3 / 8} M_{x}^{\frac{2 \nu-1}{8 \nu}+o(1)} d^{\frac{1}{8 \nu}}
\end{aligned}
$$


Notice that all exponents of $d$ in the above estimates on $A_{g}(d ; x), B_{g}(d ; x)$ and $C_{g}(d ; x)$ are nonpositive. Thus, since

$$
\sum_{d \mid M_{x}} 1=2^{\pi(x)}=M_{x}^{o(1)},
$$

in the summation over $d$ in these three expressions, the term with $d=1$ dominates. We obtain

$$
\begin{aligned}
& \sum_{d \mid M_{x}} A_{g}(d ; x) \leq h^{3 / 4}\left(\# \mathcal{G}_{g}(x)\right)^{-\frac{5 \nu^{2}+3 \nu-1}{8 \nu(\nu+1)}} Q_{g}(x)^{-3 / 8} M_{x}^{\frac{2 \nu+1}{8(\nu+1)}+o(1)}, \\
& \sum_{d \mid M_{x}} B_{g}(d ; x) \leq h^{3 / 4}\left(\# \mathcal{G}_{g}(x)\right)^{-5 / 8} Q_{g}(x)^{-3 / 8} M_{x}^{\frac{(2 \nu+1)^{2}}{16 \nu(\nu+1)}+o(1)}, \\
& \sum_{d \mid M_{x}} C_{g}(d ; x) \leq h\left(\# \mathcal{G}_{g}(x)\right)^{-\frac{5 \nu-2}{8 \nu}} Q_{g}(x)^{-3 / 8} M_{x}^{\frac{\nu-1}{4 \nu}+o(1)} .
\end{aligned}
$$

Unfortunately, the exponent of $d$ in $D_{g}(d ; x)$ is negative. However, if instead of (16) we use the trivial bound

$$
Q_{g}(d, x) \geq 1
$$

we derive the alternative estimate

$$
\begin{aligned}
D_{g}(d ; x) & \leq h\left(\# \mathcal{G}_{g}(d ; x)\right)^{-5 / 8} M_{x}^{\frac{2 \nu-1}{8 \nu}+o(1)} d^{-\frac{6 \nu-1}{8 \nu}} \\
& \leq h\left(\# \mathcal{G}_{g}(x)\right)^{-5 / 8} M_{x}^{\frac{2 \nu-1}{8 \nu}+o(1)} d^{-\frac{\nu-1}{8 \nu}}
\end{aligned}
$$

which we use for large values of $d$ (namely for $\left.d \geq Q_{g}(x)^{3}\right)$. Thus,

$$
\begin{aligned}
\sum_{d \mid M_{x}} D_{g}(d ; x)= & \sum_{\substack{d \mid M_{x} \\
d<Q_{g}(x)^{3}}} D_{g}(d ; x)+\sum_{\substack{d \mid M_{x} \\
d \geq Q_{g}(x)^{3}}} D_{g}(d ; x) \\
\ll & \sum_{\substack{d \mid M_{x} \\
d<Q_{g}(x)^{3}}} h\left(\# \mathcal{G}_{g}(x)\right)^{-5 / 8} Q_{g}(x)^{-3 / 8} M_{x}^{\frac{2 \nu-1}{8 \nu}+o(1)} d^{\frac{1}{8 \nu}} \\
& +\sum_{\substack{d \mid M_{x} \\
d \geq Q_{g}(x)^{3}}} h\left(\# \mathcal{G}_{g}(x)\right)^{-5 / 8} M_{x}^{\frac{2 \nu-1}{8 \nu}+o(1)} d^{-\frac{\nu-1}{8 \nu}} \\
= & h\left(\# \mathcal{G}_{g}(x)\right)^{-5 / 8} Q_{g}(x)^{-\frac{3(\nu-1)}{8 \nu}} M_{x}^{\frac{2 \nu-1}{8 \nu}+o(1)} .
\end{aligned}
$$

We now choose

$$
\nu=4 .
$$


Then, using Lemmas 7 and 8, one verifies that

$$
\begin{array}{ll}
\sum_{d \mid M_{x}} A_{g}(d ; x)=o(1) & \text { for } h \leq M_{x}^{0.140283}, \\
\sum_{d \mid M_{x}} B_{g}(d ; x)=o(1) & \text { for } h \leq M_{x}^{0.146316}, \\
\sum_{d \mid M_{x}} C_{g}(d ; x)=o(1) & \text { for } h \leq M_{x}^{0.139084}, \\
\sum_{d \mid M_{x}} D_{g}(d ; x)=o(1) & \text { for } h \leq M_{x}^{0.144092} .
\end{array}
$$

We now select

$$
h=\left\lfloor M_{x}^{0.139084}\right\rfloor
$$

(that is, the largest admissible value for which all of the above hold). Using Lemma 2, we see from the bounds (14) and (15) that the result of Theorem 1 follows.

Acknowledgments. S.V.K. gratefully acknowledges support from Russian Foundation for Basic Research grant 08-01-00208 and Program Supporting Leading Scientific Schools grant NSh-3233.2008.1. C.P. gratefully acknowledges support from NSF grants DMS-0401422 and DMS-0703850. I.E.S. gratefully acknowledges support from ARC grant DP0556431.

\section{References}

[1] E. Bach, R. Lukes, J. Shallit and H. C. Williams, Results and estimates on pseudopowers, Math. Comp. 65 (1996), 1737-1747.

[2] J. Bourgain, S. V. Konyagin and I. E. Shparlinski, Product sets of rationals, multiplicative translates of subgroups in residue rings, and fixed points of the discrete logarithm, Int. Math. Res. Notices 2008, art. ID rnn090, 29 pp. (corrigendum: ibid. 2009, no. 16, 3146-3147).

[3] D. R. Heath-Brown and S. V. Konyagin, New bounds for Gauss sums derived from $k$ th powers, and for Heilbronn's exponential sum, Quart. J. Math. 51 (2000), 221-235.

[4] H. Iwaniec and E. Kowalski, Analytic Number Theory, Amer. Math. Soc., Providence, RI, 2004.

[5] S. V. Konyagin, C. Pomerance and I. E. Shparlinski, On the distribution of pseudopowers, Canad. J. Math., to appear.

[6] S. V. Konyagin and I. E. Shparlinski, Character Sums with Exponential Functions and Their Applications, Cambridge Univ. Press, Cambridge, 1999.

[7] C. Pomerance and I. E. Shparlinski, On pseudosquares and pseudopowers, in: Combinatorial Number Theory, Proc. Integers Conf. 2007, Walter de Gruyter, Berlin, 2009, 171-184. 
[8] A. Schinzel, A refinement of a theorem of Gerst on power residues, Acta Arith. 17 (1970), 161-168.

Institute for Advanced Study

Department of Mathematics

Princeton, NJ 08540, U.S.A.

Dartmouth College

E-mail: bourgain@ias.edu

Hanover, NH 03755-3551, U.S.A.

Department of Mechanics and Mathematics E-mail: carlp@gauss.dartmouth.edu

Moscow State University

Department of Computing

Moscow, 119992, Russia

Macquarie University

E-mail: konyagin@ok.ru

Sydney, NSW 2109, Australia

E-mail: igor@ics.mq.edu.au

Received on 2\%.11.2008 